\title{
Nurse Leaders' Challenges Fighting the COVID-19 Pandemic: A Qualitative Study
}

\author{
Juliana S. Freitas, Ana L. Queiroz, Isabelle M. Bortotti, Cláudia R. Laselva, Eliseth R. Leão* \\ Hospital Israelita Albert Einstein, São Paulo, Brazil \\ Email: *eliseth.leao@einstein.br
}

How to cite this paper: Freitas, J.S., Queiroz, A.L., Bortotti, I.M., Laselva, C.R. and Leão, E.R. (2021) Nurse Leaders' Challenges Fighting the COVID-19 Pandemic: A Qualitative Study. Open Journal of Nursing, 11, 267-280.

https://doi.org/10.4236/ojn.2021.115024

Received: March 22, 2021

Accepted: May 7, 2021

Published: May 10, 2021

Copyright $\odot 2021$ by author(s) and Scientific Research Publishing Inc. This work is licensed under the Creative Commons Attribution International License (CC BY 4.0).

http://creativecommons.org/licenses/by/4.0/

\begin{abstract}
Nursing leaders play a pivotal role in managing the COVID-19 pandemic. Aiming to describe the challenges of nursing leaders and the solutions implemented to face the coronavirus pandemic in a Brazilian hospital we developed this qualitative study. Six tactical nursing leaders were recruited by convenience and responded via audio recordings to two open-ended questions: 1) What challenges have you experienced and what solutions have you implemented related to physical, human, and technological resources during the COVID-19 pandemic? and 2) What challenges have you experienced and what solutions have you implemented in relation to patient and staff safety during the COVID-19 pandemic? All audio files were transcribed, anonymized, and analyzed using inductive content analysis. We identified two main categories of challenges: the first, concerning the changes and challenges in the face of an unknown disease; the second category, consisting of an evaluation of the experiences lived by the nursing leaders. Numerous solutions were implemented in response to the challenging scenario. The technical competence and systemic view of nursing leaders were vital to face the challenges and develop solutions to ensure the quality of care provided and the safety of the workforce, patients, and families.
\end{abstract}

\section{Keywords}

COVID-19, Emerging Infectious Disease, Leadership, Nursing, Healthcare

\section{Introduction}

Nurses play a pivotal role in managing infectious epidemics affecting global health such as severe acute respiratory syndrome (SARS) in 2003 [1], Ebola virus disease in 2014 [2], Middle East respiratory syndrome coronavirus (MERS-CoV) outbreak in 2015 [3], Zika virus in 2016 [4], and the novel coronavirus (COVID-19) 
pandemic that broke out in late 2019 in China [5].

The first patient with COVID-19 in Brazil had a confirmed diagnosis on February 26, 2020, at Hospital Israelita Albert Einstein (HIAE), a philanthropic hospital located in São Paulo. Two weeks later the first diagnosis, all cases had a history of recent international travels. On March 11, 2020, the first case of local transmission was confirmed, also at HIAE, starting the rapid spread of this disease [6]. Since then government authorities, hospitals, private companies, and the third sector have been involved in planning and coordinating, in record time, the infrastructure, procedures, and resources to meet the high projected hospitalization rates. One year after the first confirmed case, the country experienced a second wave of COVID-19, surpasses 13 million confirmed cases, 330.000 deaths and was considered the epicenter of the global coronavirus pandemic. São Paulo, the most populous state of the country, was one of the worst-hit regions, with more than 2.5 million cases and 77.000 deaths at the time [7].

The pandemic has highlighted the crucial role of nurses in health care services. Nurses work on the frontline of the health care system, provide infection control education, and help reduce the spread of incorrect information about the pandemic [8]. Though, in order for this to be effective, nursing leaders must work together in a collaborative manner following the recommendations of regulatory bodies at national and international level and based on the state-of-the-art scientific evidence available.

Nursing leaders oversee the development and training of staff with respect to institutional protocols for patients with suspected or confirmed COVID-19, ensuring that all patients receive safe, individualized, high-quality care. Additionally, they must ensure the protection of frontline nurses, closely monitoring their well-being and mental health [9], all of which in a unique, challenging times of great uncertainty when developing solutions quickly while looking at the big picture and the consequences at the small picture through a social-political lens is an imperative [10].

The central role of frontline nurses, their heroism and their experiences during COVID-19 pandemic gained great visibility and has permeated dense discussions and investigations worldwide. Nonetheless, little attention has been paid to nursing leaders and their experiences. These professionals, although not working directly with patients, have faced single challenges to mobilize system and individual level strategies and resources to support staff in managing pandemic-related issues [11].

An initial study on the impact of COVID-19 on nursing leadership in the United States, conducted in July 2020 by the American Organization for Nursing Leadership and Joslin Marketing with nearly 2000 nurse leaders, revealed the magnitude of challenges, which included lack of a playbook, shortage of personal protective equipment (PPE) and supplies, ever-changing information, changes in cultural dynamics, and financial impact on staff. Almost 15\% of the respondents, stated they were not prepared for a future pandemic or surge [12]. To the International Nursing Council, health organizations must capitalize on nursing 
leadership in order to get through this health crisis and learn from the COVID-19 pandemic to be prepared for the future [13].

The challenges of tactical-level nursing leaders and the solutions implemented to face the COVID-19 pandemic in Brazilian organizations is currently an open question that we seek to help understand with this study.

\section{Materials and Methods}

\subsection{Study Design and Participants}

This qualitative study was conducted with tactical-level nursing leaders from Hospital Israelita Albert Einstein, a high-complexity, 627-bed philanthropic hospital located in São Paulo, Southeast region of Brazil, which is the national reference center for treatment of COVID-19 patients. Tactical-level nursing leaders was defined as those nursing professionals that serve as the hub for both the strategic and operational levels and who are tasked with determining how the operational level can best achieve the institutional goals outlined by the organization.

All eight nursing leaders who were delegated to the HIAE crisis committee were invited to participate in the study, six of whom (hereafter referred to as P1 P6) were included. The study invitation was sent by email, containing the objectives, complete guidance on the interview procedures and the use of WhatsApp for communication and deadlines for response. One leader who believed to have an operational role declined the invitation to participate and another leader working in operational level was excluded from the study.

The study was conducted in accordance with Brazilian law for research with human beings and the Declaration of Helsinki. The study was approved by the National Commission for Research Ethics (Presentation Certificate for Ethical Appreciation number 3.974.416). Written informed consent was obtained from all participants.

\subsection{Procedures}

Interviews were conducted in May 2020 using the Mobile Experience Sampling Method (MESM), which gathers individuals' insights into their feelings and/or thoughts over a period of days using mobile technology [14]; in this study, we used the instant messaging app WhatsApp.

Participants responded via audio recordings to the following broad data-generating questions, developed on the basis of an extensive literature review and the researchers' empirical experiences: 1) What challenges have you experienced and what solutions have you implemented related to physical, human, and technological resources during the COVID-19 pandemic? and 2) What challenges have you experienced and what solutions have you implemented in relation to patient and staff safety during the COVID-19 pandemic? Each question was sent out individually to participants by the principal investigator with a 72-h interval between questions. The audio response time was unlimited so partici- 
pants could share their experience in fullness.

The experience-sampling method and instant messaging application used in the study were carefully analyzed and their selection was justified by the need to facilitate data collection in a timely manner during the challenging times of a pandemic, when the personal and professional routines of participants were significantly affected, with heavy workloads and unpredictable work demands.

\subsection{Data Analysis}

All audio recording files were transcribed, anonymized, and analyzed using inductive content analysis [15] with the goal of providing new insights, and a representation of facts pertaining to challenges and solutions to face the COVID-19 pandemic. Two authors independently prepared and organized the data, openly coded transcripts and formulated the categories and subcategories [16]. The findings were then compared and discussed until all authors agreed on the thematic categories, subcategories and example quotations.

\section{Results}

The sample consisted of six tactical-level nursing leaders who are characterized in Table 1.

\subsection{The Challenges}

Two main categories and eight subcategories related to the challenges emerged from discourse analysis of the nursing leaders' experiences (Table 2).

The first thematic category, "changes and challenges in face of the unknown COVID-19 pandemic" comprised five subcategories related to preparation of human resources, physical structure, equipment and supplies, care protocols and information for the response to the pandemic. Participants experienced changes and challenges in the different areas of the organization. The planning and implementation of solutions took place in an uncertain scenario, based on institutional projections, which considered, among other factors, the behavior of the virus in countries where the pandemic had was already underway and both the failed and successful experiences in those countries.

Table 1. Participant characteristics.

\begin{tabular}{ccccc}
\hline Nursing leader & Age (yrs) & Gender & $\begin{array}{c}\text { Leadership } \\
\text { experience }(\mathrm{yrs})\end{array}$ & Current leadership position \\
\hline P1 & 34 & Female & 5 & Processes and operations coordinator \\
P2 & 42 & Female & 7 & Nursing coordinator \\
P3 & 50 & Male & 15 & Planning and control specialist \\
P4 & 39 & Female & 3 & Patient experience and safety manager \\
P5 & 35 & Female & 8 & Patient assistance manager \\
P6 & 37 & Female & 5 & Patient flow manager
\end{tabular}


Table 2. Thematic categories and subcategories related to the challenges.

1. Changes and challenges in face of the unknown COVID-19 pandemic
A Human resources preparedness
B Physical structure preparedness
C Equipment and supplies preparedness
D The adequacy of patient care
E Information updating

2. Appraisal of nursing leaders' experiences
A Positive aspects
B Negative aspects
C Lessons learned

To ensure adequate staffing, nursing leaders had to resort to emergency hiring: "The emergency hiring of multiple health care personnel to work in critical care settings was both an action and a challenge. Over 400 health care workers were hired in one month. That was a very big challenge, and the different units, in collaboration with Human Resources area, took several actions, including mass interviews, application of quick tests, admission, and integration of health care professionals, all within two days." (P5)

The reallocation of staff to other units was also a necessity: "We saw health care workers who had to completely change their scope of practice and dedicate themselves to a new demand." (P1)

The training of all staff in the new institutional flows and care protocols was compulsory and performed "in record time, to handle all demands, which required fast deliveries." (P4)

Staff safety was a challenge for the nursing leaders since they started confronting the pandemic: "A major concern was hospital-acquired COVID-19 infection." (P4) One leader said: "We also spoke a lot about the best practice in personal protective equipment (PPE) use, the need to care for one another to ensure access to PPE, and that no one lacked any PPE." (P5)

Despite efforts to protect health care workers at the organization, cases of community transmission have been promptly detected in Brazil, increasing the number of cases among health care workers: "When the spread of the disease accelerated in late March 2020, we were faced with another challenge, which was a lack of staff, not just because of hospital-acquired infection, but because of the community transmission. For example, the intra-hospital transport team numbers 85 staff and, in a single day, 57 workers took a leave of absence for COVID-19." (P6)

The hospital's physical structure also required changes to meet the high projected hospitalization rates. Expansions and adjustments in the existing facilities were completed in a short time. "The first challenge was the search for expansion sites." (P5); "We turned approximately 400 beds into hybrid beds. Which 
means that these beds have all the infrastructure to provide care to patients in intensive care, semi-intensive care, or standard supportive care." (P6); "We faced the challenge of adapting the facilities and installed a ventilation system that generated negative pressure in the rooms." (P4)

In this context, it was crucial to purchase and stock myriad medical equipment and supplies that, at times, were currently already unavailable at the national and international market. New commercial partners were selected, and purchase flows were rearranged in view of the organizational agility required. "We could not find the amount of equipment needed in the same model as the equipment we previously used here at the hospital, which had already been regulated/approved, because they were not available in those quantities. The team now had to deal with supplies and equipment that were different from the usual ones." (P3); "We had to pick and choose new commercial partners, new suppliers. That was also a big challenge, because we had to get familiar with the equipment, the structure of the companies, their capacity to deliver." (P3)

The lack of standardized equipment and supplies at the hospital required the development of new quality verification processes. Medical gowns and alcohol-based hand rubs were critical supplies during the pandemic: "The domestic producers and the Hospital Infection Control Service began testing gowns, evaluating their thickness, and ensuring they complied with the minimum safety standards, so that they could be approved and eventually purchased. Alcohol-based hand rubs was another critical item, and some interesting solutions were found because we could no longer source alcohol-based hand rubs refills for use with the hospital's standardized wall dispenser, suppliers were asked to develop a dispenser so we could use the tabletop hand sanitizer refills, which were widely available at the time, on the wall dispensers." (P4)

The institutional care protocol for patients with suspected or confirmed COVID-19 infection was developed with a focus on providing access to timely quality and safe care: "The first challenge came up precisely at this time, to develop, create guidelines that were safe enough for us to continue providing excellent care and delivering adequate results in the response to this pandemic." (P4) As part of this protocol and to optimize safety, other processes have been reviewed: "Coordinators were responsible for determining, in each of their units, what the actual need was for a patient to have a companion. There were exceptions to the suspension of visiting. Surely, we allowed family members to visit patients with terminal illness or end-stage disease. Family members could say goodbye to patients, but always wearing the appropriate PPE, and they were supervised while putting on PPE." (P5) Such imposed restrictions could potentially adversely affect the patient's experience and his/her mental health. Nursing leaders praised the innovative work of psychologists and nursing staff: "The Psychology staff took a very important and interesting action in bringing tablet computers to allow family members to communicate with these patients. The Nursing staff also played a very significant role in involving the family, and they were often in contact with family members to provide them with an updated medical report of 
the patient." (P5) One of the leaders highlighted that "Patient and staff safety has always been a core value for our organization and has not been neglected at any time." (P3)

Care for non-COVID-19 patients who were seeking care for other health issues followed a specific protocol. Their safety was equally challenging: "Another challenge is to ensure the safety of patients who were admitted to non-cohort units and try our best to preserve these patients, preventing in-hospital contamination and that they acquired the disease during their hospitalization." (P5)

Because COVID-19 is a new disease, an overwhelming amount of information is published daily, nationally and internationally, which requires that nursing leaders and the organization keep up with the constant flow of new medical information, based on reliable sources: "The challenge is to study frequently, read newly published research every day, review our guidelines." (P4)

An up-to-date institutional communication was crucial to the success of COVID-19 protocols: "Keeping the team well informed and being able to translate the actions, directions, with solid sources, for people to feel safe." (P6) The large number of workers that had to receive the information was a challenge: "Getting the information to the end-user is a big challenge. There is a lot of information that we have to pass on to the operational team. Today, there are nearly 700 workers in the inpatient department, 650 of whom are front-line health-care workers." (P5)

A few months after they had started facing the pandemic, nursing leaders listed not only the main changes and challenges, but also acknowledged both the positive and negative aspects and the lessons learned, which were grouped into the second thematic category "appraisal of nursing leaders' experiences".

One of the positive aspects identified by nursing leaders was the opportunity to get to know new partners, new resources, and new forms of interpersonal relationship at the organization: "We have met some new good partners in this process." (P3); "One very important characteristic is that we've learned to use technological resources in our communications." (P6); "I have met a lot of people, acquired a lot of knowledge, not only about nursing management, but also about information technology issues." (P2); "We have heard about the importance of hand hygiene for years, but I think that such simple and invaluable action carries huge importance during this pandemic. With COVID-19, there was a significant increase in infection control, quality and safety activities. And with COVID-19, we learned to respect each other more. Besides taking care of myself, I respect others, I care for others." (P4)

The nursing leaders' experiences were also negatively impacted by heavy workloads, new demands, and a new reality at home and with their families as a result of the COVID-19 pandemic. "I usually say we've always worked a lot, but I've never worked so much in my life. This pandemic has challenged us to think in a completely different way, to act with great speed. In addition to the speed, we had to pore over daily studies of materials produced about various issues, including transmission, care, safety of support and operational processes, levels of 
evidence, because things are very dynamic. It is a pressure that I have never experienced anything close or similar to that." (P6); "We know how much it impacts everyone's lives, we're affecting the lifestyle of our co-workers too, changing work units, for example, the profile of the patients they service." (P1)

The lessons learned by the nursing leaders during the COVID-19 pandemic extend beyond technical or operational issues: "I think this is a great moment, it is more than a professional experience, it is a life experience and I have no doubt whatsoever about that, of all the lessons we've learned and will learn within this scenario." (P6) In this context, human and teamwork and collaboration issues stood out: "The learning is very clear, how much we managed to work in a collaborative manner to train different teams." (P6); "There were very important learnings, and acknowledging how much we depend on others, how by caring for others I am doing much for myself; it has been a life lesson of this pandemic." (P6)

\subsection{The Solutions}

The discourse of the interviewed nursing leaders also showed numerous solutions implemented to face the changes and challenges imposed by the COVID-19 pandemic. A summary of the solutions is shown in Table 3.

Table 3. Solutions implemented by nursing leaders to face the challenges imposed by the COVID-19 pandemic.

Nursing leader
$\begin{aligned} & \text { Human resources preparedness } \\ & \text { Health professionals received rapid training, focused on the care of critically ill } \\ & \text { patients, care for invasive mechanical ventilation, the mobilization of critically ill } \\ & \text { patients, the administration of vasoactive drugs and sedations. They were also } \\ & \text { trained in the use of new equipment, such as multiparameter monitors. }\end{aligned}$
P6 $\quad \begin{aligned} & \text { We did an immediate mass training of the entire hospital cleaning staff, in-hospital } \\ & \text { patients. }\end{aligned}$

Physical structure, equipment and supplies preparedness

Equipment acquisitions were made through permanent purchases and, in some cases, through temporary leasing.

We started to have daily meetings to monitor the acquisition process, since it was based on institutional projections. Therefore, if the context of the pandemic changed, it might be necessary to change the quantity and characteristics of the equipment being purchased.

We purchased shelving units for individual PPE storage for all hospital areas. We have implemented carts containing gloves, cleaning product, and garbage space for PPE doffing. These carts are mobile, so professionals can use it close to the purge areas to feel safer when cleaning their glasses or face shield.

The adequacy of patient care

P5 We have implemented clinical indicators to estimate the complexity of patients with confirmed COVID-19 based on the clinical deterioration score. 


\section{Continued}

We have five large electronic panels to manage all patients with suspected or confirmed COVID-19, ranging from the moment of testing, result analysis, patient admission, to discharge or death. We have information in real time, all the time, from the entire hospital.

We institute flags in the COVID-19 PCR test orders and link them to patients' medical records, to ensure the correct admission of patients to the cohort units.

The intrahospital patient transport system was changed and started to be mandatorily performed with two professionals, no matter if the request is for patients with suspected or confirmed COVID-19. One of the professionals performs all the contacts with the patient and with the transport device, such as a stretcher or wheelchair. The other professional is responsible for the transport directions, lift operation and door opening, exclusively.

We have a control list of professionals to monitor contact with patients with

P2 confirmed COVID-19 and this information is available in the medical record. Therefore, all professionals who enter the patient's room to provide care must insert their name on the list.

Information updating

Practices such as participatory leadership, the crisis room, the daily safety huddle, gave us much more security and support to pass on information to those who are working on the front line. and Marketing areas and by the leaders.

\section{Discussion}

In any crisis, leaders have two equally important responsibilities: solving the problem at hand and preventing it from occurring again; both responsibilities are confronted with short- and long-term challenges [17]. Pervasive throughout the discourse of Brazilian nursing leaders, one can perceive that dealing with the COVID-19 pandemic was marked by numerous challenges related to changes and to ensuring the quality of care, safety, and well-being of patients, families, and the workforce. These challenges have been exacerbated by the unprecedented nature of the emergency, which the leaders had never before experienced in their careers and to which their training, centered on patient care and hyperspecialization [18], could not prepare them. Even though disease scenarios and coping strategies can be very different globally, previous studies have shown that other leaders have faced similar challenges [19] [20].

The first COVID-19 case in Brazil was diagnosed and treated at the hospital that hosted the study, which anticipated the arrival of the disease in the country and had, at the time, a crisis committee and a contingency plan [21]. The tactical leaders interviewed, guided by the institutional strategic leadership, prepared for the worst-case scenario, which required solid and up-to-date guidelines and protocols, as well as guidance and involvement from the operational level.

Healthcare system infrastructures in all countries have been overwhelmed by the pandemic, exposing their limited capacity to provide acute care, which is centered on the availability of human resources and medical equipment and 
supplies essential to the safety of patients and health care workers alike [22] [23] [24] [25]. In this scenario, it was critical that nursing leaders operated from an excellence-based model and learned to deal with fast, and often uncertain, decision making, understanding the working environment and establishing clearly the roles and responsibilities of each team member [19] [26]. All decisions, both big and small, needed to represent and prioritize patient and family needs [19].

The nursing leaders interviewed acknowledged that the safety of patients, companions, and health care workers has always been at the forefront of the hospital and has pervaded all planning and actions. Ensuring adequate staff skill mix required that nursing leaders provide not only adequate staffing levels but also education and training to maximize the ability of each nurse to deliver quality safe care to a large number of patients over a long period of time [27]. The implantation of an "Intelligence Center" in the hospital, formed by a group of researchers, assisted leaders to keep updated on the national and international literature and guidelines related to the coronavirus. These researchers were responsible for continuously synthesizing the main evidence published daily in high-impact journals and by public health organizations and refer them to the leaders to support their activities and decisions. Evidence-based training was extensively provided, and it requested from the leaders, beyond technical and scientific competence, specific people-management skills for dealing with and overcoming fear among nurses and the unease and anxiety caused by the reallocation of staff to areas they felt unprepared to practice.

Updated patient, companion, and staff flows were some of the changes made with a focus on safety. The promptness for change highlighted by the leaders in the interviews may have been influenced by the experience of the nursing staff, but also because the organization has been accredited by the Joint Commission International (JCI) since 1999 and has a "Catastrophe Plan". One of the JCI standards focuses on the ability of the organization to address situations that extend beyond normal hospital routine and demand the implementation of alternative service flows. Moreover, the organization is on the journey to Magnet $^{\circ}$ recognition for nursing excellence and its ability to deliver excellent results to patients. Transformational leadership is an important component of this model and has been associated with high-quality care delivered to COVID-19 patients [26].

Although HIAE is a nongovernment philanthropic organization, it has supported state and local public health through the administration of 16 public primary care units and two high-complexity public hospitals. In 2020, the organization was responsible for the implementation and maintenance of a field hospital in the city. In this context, much of the planning and solutions described by the leaders in this study were applied and benefited underserved communities and the National Healthcare System.

Unfortunately, the multiple social and economic issues and the vulnerability of health systems observed across Brazil have led to different experiences in res- 
ponding to COVID-19. Qualitative study conducted in the southern region of the country with nurses who held leadership positions in health organizations, educational institutions and the Regional Nursing Council at the time of the pandemic revealed that these leaders experienced several challenges related to deficit of financial resources for the management and operation of the system during the crisis, added to the unpreparedness of nursing staff, poor working conditions, work overload and high lethality of nursing professionals [24].

During the pandemic, nurses gained visibility in the media and were often portrayed as angels or superheroes, constructs that are part of the social imaginary associated with nursing, but which are far removed from the reality that emerged from the nursing leaders' discourse in this study. Yet even if these superhuman attributes are bestowed on nurses as a gesture of recognition, they are not sufficient to ensure that nurses provide quality care in face of the challenges imposed by the pandemic. This requires, above all else, knowledge, skill, critical thinking, empathetic communication, investment of time and effort, commitment to patients [28], and compassionate approaches to leading colleagues and acknowledging your own and others' wellbeing needs [29].

Despite numerous challenges, nursing leaders showed great capacity for adaptability, creativity and innovation to design and implement solutions, and improve the experience of the staff, patients and their families. Even though they were often overworked and stressed, nursing leaders felt empowered by recognizing their capabilities, which were put to the test during the times of crisis and noticed the development of new skills. Moreover, personal issues were also involved, and as humans under extreme pressure, nursing leaders recognized the importance of care and self-care and the transformative experience they will take with them for life.

The study findings should be placed within the context of its limitations. First, the study design precludes generalizing to other settings or situations. Second, the study synthesizes the experience of a group of tactical leaders from an international accredited organization with the financial resources required for investing in COVID-19 response efforts, which provided nursing leaders with the optimal conditions to perform their job.

Considering the multiple waves of coronavirus experienced to date and the global susceptibility to new outbreaks of infectious diseases, leaders and health care workers in all settings need to be adequately prepared to deal with these complex epidemiological challenges and mitigate their impacts.

Some authors have discussed that, the future of nursing leadership will require a fundamental improvement in leadership style, rooted in a willingness to act in multidisciplinary and interprofessional collaborative decision-making, decentralize authority, empower a broader network and build processes that augment the leader's capacity to act according to the needs of the organization, staff, patients and family [30] [31]. Our findings show that the future has already arrived for these leaders along with the COVID-19 pandemic. 


\section{Conclusion}

The COVID-19 pandemic imposed numerous personal, professional, and organizational challenges to tactical-level nursing leaders, which were overcome through knowledge, dedication, teamwork, resilience, and institutional support. Nursing leaders played a key role in facing the challenges and developing solutions that guarantee the quality of care provided and the safety of the workforce, patients and their families during the pandemic, which is something to be acknowledged and valued. The findings of this study can serve as the basis for reviewing and adjusting institutional plans, support and training protocols, and policies adopted during the COVID-19 pandemic to incorporate the lessons learned and improve the actions, results, and experience of all personnel involved in the present and future challenges.

\section{Conflicts of Interest}

The authors declare no conflicts of interest regarding the publication of this paper.

\section{References}

[1] Shih, F.-J., et al. (2009) Surviving a Life-Threatening Crisis: Taiwan's Nurse Leaders' Reflections and Difficulties Fighting the SARS Epidemic. Journal of Clinical Nursing, 18, 3391-3400. https://doi.org/10.1111/j.1365-2702.2008.02521.x

[2] Sagar, P. (2015) Nurses Leading the Fight against Ebola Virus Disease. Journal of Transcultural Nursing, 26, 322-326. https://doi.org/10.1177/1043659615574326

[3] Choi, J. and Kim, K. (2016) Crisis Prevention and Management by Infection Control Nurses during the Middle East Respiratory Coronavirus Outbreak in Korea. American Journal of Infection Control, 44, 480-481. https://doi.org/10.1016/j.ajic.2015.10.032

[4] Wilson, A. and Nguyen, T. (2017) The Zika Virus Epidemic: Public Health Roles for Nurses. OJIN: The Online Journal of Issues in Nursing, 22, 4.

[5] International Council of Nurses (2020) COVID-19 and the International Supply of Nurses. Geneva.

[6] Teich, V., et al. (2020) Epidemiologic and Clinical Features of Patients with COVID-19 in Brazil. Einstein, 18, eAO6022. https://doi.org/10.31744/einstein_journal/2020AO6022

[7] Ministério da Saúde (2020) Painel de casos de doença pelo coronavírus 2019 (COVID-19) no Brasil. https://covid.saude.gov.br

[8] Choi, K., Skrine, J. and Logsdon, M. (2020) Nursing and the Novel Coronavirus: Risks and Responsibilities in a Global Outbreak. Journal of Advanced Nursing, 76, 1486-1487. https://doi.org/10.1111/jan.14369

[9] American Nurses Association (2020) Coronavirus Disease (COVID-19). What Nurses Need to Know.

https://www.nursingworld.org/practice-policy/work-environment/health-safety/dis aster-preparedness/coronavirus

[10] Salvage, J. and White, J. (2019) Nursing Leadership and Health Policy: Everybody's Business. International Nursing Review, 66, 147-150.

https://doi.org/10.1111/inr.12523 
[11] Hofmeyer, A. and Taylor, R. (2021) Strategies and Resources for Nurse Leaders to Use to Lead with Empathy and Prudence So They Understand and Address Sources of Anxiety among Nurses Practising in the Era of COVID-19. Journal of Clinical Nursing, 30, 298-305. https://doi.org/10.1111/jocn.15520

[12] Joslin, D. and Joslin, H. (2020) Nursing Leadership COVID-19 Insight Survey: Key Concerns, Primary Challenges, and Expectations for the Future. Nurse Lead, 18, 527-531. https://doi.org/10.1016/j.mnl.2020.10.002

[13] International Council of Nurses (2020) ICN Highlights Top Priorities to Beat COVID-19. https://www.icn.ch/news/icn-highlights-top-priorities-beat-covid-19

[14] Hedstrom, A. and Irwin, M. (2017) Mobile Experience Sampling Method (MESM). In: Matthes, J., Ed., The International Encyclopedia of Communication Research Methods, John Wiley \& Sons, Hoboken, 2048.

[15] Krippendorff, K. (2013) Content Analysis. An Introduction to Its Methodology. 3rd Edition, SAGE, California.

[16] Elo, S. and Kyngäs, H. (2008) The Qualitative Content Analysis Process. Journal of Advanced Nursing, 62, 107-115. https://doi.org/10.1111/j.1365-2648.2007.04569.x

[17] Gates, B. (2020) Responding to Covid-19-A Once-in-a-Century Pandemic? The New England Journal of Medicine, 382, 1677-1769.

[18] Geremia, D., et al. (2020) COVID-2019 Pandemic: Nursing Training and Performance for the Unified Health System. Enfermagem em Foco, 11, 40-47.

[19] Aquilia, A., et al. (2020) Nursing Leadership during COVID-19: Enhancing Patient, Family and Workforce Experience. Patient Experience Journal, 7, 136-143. https://doi.org/10.35680/2372-0247.1482

[20] Rosa, W., Schlak, A. and Rushton, C. (2020) A Blueprint for Leadership during COVID-19. Nursing Management, 51, 28-34. https://doi.org/10.1097/01.NUMA.0000688940.29231.6f

[21] Hospital Israelita Albert Einstein (2020) Nota sobre o novo coronavírus. https://www.einstein.br/sobre-einstein/sala-imprensa/press-release/nota-sobre-nov o-coronavirus

[22] Emanuel, E., et al. (2020) Fair Allocation of Scarce Medical Resources in the Time of Covid-19. The New England Journal of Medicine, 382, 2049-2055. https://doi.org/10.1056/NEJMsb2005114

[23] Estalella, G., Zabalegui, A. and Guerra, S. (2020) Management and Leadership of Nursing Services in the Emergency Plan for the Pandemic COVID-19: The Experience of the Clinic Hospital of Barcelona. Enfermeria Clinica, 31, S12-S17. https://doi.org/10.1016/j.enfcli.2020.05.002

[24] Geremia, D., et al. (2020) 200 Years of Florence and the Challenges of Nursing Practices Management in the COVID-19 Pandemic. Revista Latino-Americana de Enfermagem, 28, e3358. https://doi.org/10.1590/1518-8345.4576.3358

[25] Liu, Q., et al. (2020) The Experiences of Health-Care Providers during the COVID-19 Crisis in China: A Qualitative Study. The Lancet Global Health, 8, e790-e798. https://doi.org/10.1016/S2214-109X(20)30204-7

[26] Erickson, J., Johnson, S. and Blanchfield, B. (2020) Using Magnet Model Components at a COVID-19-Positive Field Hospital. JONA: The Journal of Nursing Administration, 50, 435-437. https://doi.org/10.1097/NNA.0000000000000912

[27] Shanafelt, T., Ripp, J. and Trockel, M. (2020) Understanding and Addressing Sources of Anxiety among Health Care Professionals during the COVID-19 Pandemic. JAMA, 323, 2133-2134. https://doi.org/10.1001/jama.2020.5893 
[28] Stokes-Parish, J., et al. (2020) Angels and Heroes: The Unintended Consequence of the Hero Narrative. Journal of Nursing Scholarship, 52, 462-466.

https://doi.org/10.1111/jnu.12591

[29] James, A. and Bennett, C. (2020) Effective Nurse Leadership in Times of Crisis. Nursing Management (Harrow), 27, 32-40. https://doi.org/10.7748/nm.27.6.12.s11

[30] Rosser, E., et al. (2020) The Need for Visible Nursing Leadership during COVID-19. Journal of Nursing Scholarship, 52, 459-461. https://doi.org/10.1111/jnu.12587

[31] Lobdell, K.W., et al. (2020) Improving Health Care Leadership in the Covid-19 Era. NEJM Catalyst. 